\title{
CONTENT AND COMPOSITION OF THE MUCOPROTEIN FRAC- TION OF HUMAN SERUM (SEROMUCOID) IN DISEASE, WITH SPECIAL REFERENCE TO HEMATOLOGIC DISORDERS ${ }^{1}$
}

\author{
By ELLY MOSCHIDES, ${ }^{2}$ MARIO STEFANINI, ${ }^{3}$ SERGIO I. MAGALINI, ${ }^{4}$ AND \\ STEN A. KISTNER 5 WITH THE TECH NICAL ASSISTANCE OF ROSE MELE \\ (From the Joseph Stanton Memorial Laboratories, St. Elizabeth's Hospital, and the Department \\ of Medicine, Tufts University School of Medicine, Boston, Mass.)
}

(Submitted for publication November 13, 1956; accepted September 12, 1957)

Significant alterations of content, distribution, electrophoretic mobility and, possibly, composition of carbohydrate-containing proteins in many diseases have been described by both chemical and electrophoretic methods. From a summary of the pertinent observations in the literature (Table I), it may be concluded that the carbohydrate content of proteins is high in many disease states; also, that the electrophoretic distribution of polysaccharides or their chemical precipitation with the various protein fractions may often be abnormal. Thus, the study of carbohydrate-containing proteins of serum is a potentially important and significant tool in the detection of basic molecular alterations, which may be significant in the understanding of the pathogenesis of disease.

We have attempted in a number of disease states a systematic study of the content and in the chemical composition of one type of carbohydratecontaining proteins, the mucoproteins. Because of the major interest of our Institution and our previous work on the subject particular emphasis was directed to hematologic disorders. The serum mucoprotein fraction was selected for this study because: a) it shows significant and wide variations in disease (Table $I$ ) ; b) fairly reliable techniques are available for its isolation and for the analysis of some of its constituents. Total muco-

\footnotetext{
1 Supported by a grant from the Massachusetts Cancer Society and grant-in-aid H-2132 from the National Institute of Health, United State Public Health Service.

2 Research Assistant, Joseph Stanton Memorial Laboratories.

${ }^{3}$ Established Investigator, American Heart Association.

4 Formerly Damon Runyon Cancer Research Fellow. Present address: Cancer Research Foundation, Boston.

5 Fellow of the American-Scandinavian Foundation. Present address: Department of Biochemistry, Nobel Institute, Stockholm, Sweden.
}

proteins, mucoprotein-bound hexoses and hexosamine were studied. For comparison, values for total protein, total hexosamine and total nonglucosamine protein-bound hexoses were also obtained for all sera. Our work was handicapped by the present confusion in classification and definition of the polysaccharide-containing proteins. These have been divided by Meyer (1) into glycoproteins and mucoproteins. The two types appear similarly constituted with a mucopolysaccharide held to a protein moiety by a firm chemical bond. However, in mucoproteins, the degree of alcohol solubility is higher and the hexosamine content is more than 4 per cent weight. In glycoproteins, hexosamine represents less than 4 per cent. In this study the definition of mucoprotein, as suggested by Winzler, Devor, Mehl, and Smyth (2), was applied to that fraction of serum proteins soluble in perchloric acid solution, and precipitable by phosphotungstic acid. The fraction we studied appeared comparable to the seromucoid of Rimington (3) and the seroglycoid of Hewitt (4). Since we considered as mucoproteins a group of proteins of serum on criteria of solubility alone, only relative conclusions were possible. In particular, our findings have no bearing on the exact nature of mucoproteins and on their relationship to other carbohydrate-containing proteins.

\section{METHODS}

Total serum protein (T.P.) was determined by the method of Gornall, Bardawill, and David (5), which employs the biuret reaction; total non-glucosamine, protein-bound hexoses (T.Ph.) by the procedure of Sheppard and Everett (6) ; total hexosamine (T.Ha) by the method of Rimington (3) ; (d) serum mucoproteins (T.Mp.) were separated as described by Winzler and co-workers (2). This method is based on the precipita- 
TABLE I

Serum total hexoses, protein-bound hexoses, total hexosamine and mucoprotein in disease: $A$ summary*

Total hexoses

Protein-bound hexoses

Protein-bound hexoses: total protein ratio

Total hexosamine

Mucoprotein (seromucoid)

Mucoprotein-bound hexoses
Elevated in advanced tuberculosis $(21,22,16)$, sarcoidosis and cancer $(22,16)$, rheumatic fever in relapse (9), rheumatoid arthritis (15), diabetes mellitus with complications (14), disseminated malignancies $(23,24)$, leukemia, lymphoma, chronic infections, colitis, acute glomerulonephritis, prostatic hypertrophy (24).

Elevated in carcinoma, benign tumors, arthritis, bacterial infections (17), disseminated malignancies $(25,26)$, tuberculosis $(27,28)$. Hexoses in serum albumin fraction: elevated in normal pregnancy (29), more so in carcinoma (17) and rheumatic fever (9).

In multiple myeloma, the pathological protein contains maximum amount of polysaccharides (30). In pneumonia, polysaccharides are high in all electrophoretic fractions (31).

Elevated in rheumatoid arthritis, in relation to severity of symptoms (32).

Elevated in rheumatic fever (9), pneumonia (33), carcinoma. Hodgkin's disease, leukemias, rheumatoid arthritis, periarteritis nodosa, rheumatic fever, chronic infection, pneumonia, myocardial infarction (34), lupus erythematosus (35), gout, cirrhosis of the liver, hepatitis (18).

Hexosamine is highest in the gamma globulin fraction of patients with lupus erythematosus (36). In other diseases, the $\alpha_{1}$-globulin contains most of the increased hexosamine (18).

Elevated in diabetes mellitus with complications (14), myocardial infarction (37), carcinoma and pneumonia (19), rheumatic fever (38), trauma (12). Decreased in liver disease, some endocrine dysfunctions, nephrosis (12).

Elevated in rheumatoid arthritis (15).

* The terminology used in this table is in agreement with that used in the text.

tion of all proteins by perchloric acid and subsequent isolation of mucoproteins which are insoluble in phosphotungstic acid. ${ }^{6}$ After isolation, the following components of mucoproteins were determined: a) protein moiety (T.Mp.) ; b) hexoses (Mh.) ; c) hexosamine (Mha.). Mean values and standard deviation were calculated for each value according to the formula:

$$
\sigma=\sqrt{\frac{\sum \mathrm{i}(\overline{\mathrm{x}}-\mathrm{xi})^{2}}{\mathrm{n}}},
$$

where $\bar{x}$ represented the mean, $x i$ the $i^{\text {th }}$ individual determination, and $\mathrm{n}$ the number of determinations. The significance of our results was further evaluated by means of the formula (7):

$$
\mathrm{t}=\frac{\overline{\mathrm{X}}_{1}-\overline{\mathrm{X}}_{2}}{\mathrm{~S}_{1-2}} \sqrt{\frac{\mathrm{N}_{1} \cdot \mathrm{N}_{2}}{\mathrm{n}_{1}+\mathrm{n}_{2}}}
$$

${ }^{6}$ Winzler and co-workers (2) have indicated that dilution of serum and final molarity of perchloric acid are essential to optimal precipitation of a constant amount of mucoprotein. They have advised a concentration of $0.6 \mathrm{M}$ perchloric acid. Results indicated in Tables II and III show that dilution and molarity of perchloric acid were of relatively little bearing on the final yield of mucoproteins. Thus, a molarity of $0.24 \mathrm{M}$ was used in the course of this study, a figure which seemed to assure the complete precipitation of the total protein fraction with a minimum amount of reagent. The serum was diluted 1:5 with perchloric reagent as suggested by Winzler. where $S_{1-2}$ equalled

$$
\sqrt{\frac{\Sigma \mathrm{x}_{1}^{2}+\Sigma \mathrm{x}_{2}^{2}}{N_{1}+N_{2}-2}}
$$

In the latter formula, $\overline{\mathrm{X}}_{1}$ indicated the mean of normal values; $\bar{X}_{2}$, the mean of the values being investigated; $N_{1}$, the number of samples in the normal group; $\mathrm{N}_{2}$, the number of samples in the group investigated; $x_{1}$ the square root of the differences of each sample in the normal group from the mean value; $x_{2}$ the same value for the group investigated. The significance of $t$ values was judged on the basis of a standard table (8).

\section{Materials (Table IV)}

Eight healthy individuals and 111 patients were included in this study. Eighty-eight patients represented cases seen in the wards of our Institution. Serum for an additional twenty-three patients was obtained through the courtesy of Drs. Jean Dausset (Centre National des Transfusions Sanguines, Paris, France), James Monto (Henry Ford Hospital, Detroit, Michigan) and Donat Cyr (Lahey Clinic, Boston, Massachusetts). These samples may be recognized in all tables since they are indicated by number, rather than by patient's initials; they were included in the series to verify the reliability of results against possible errors in diagnosis. Serum from local patients was promptly frozen and stored at $-20^{\circ}$ C. until used. Sera from other areas were sent by air in containers filled with dry ice. In each in- 
TABLE II

Effect of the dilution of serum on the recovery of mucoproteins and their composition: $A$ representative experiment

\begin{tabular}{|c|c|c|c|c|c|c|}
\hline & & & \multirow{2}{*}{$\begin{array}{l}\text { Weight } \\
\text { of dry } \\
\text { precipitate }\end{array}$} & \multicolumn{3}{|c|}{ Composition of mucoproteins } \\
\hline & & & & Protein & Hexoses & Hexosamine \\
\hline & Normal serum dilution & $\begin{array}{l}1: 1 \\
1: 2 \\
1: 5\end{array}$ & $\begin{array}{c}m g . \% \\
83 \\
83 \\
82\end{array}$ & $\begin{array}{c}\text { mg. } \% \\
80 \\
80.9 \\
82\end{array}$ & $\begin{array}{c}m g . \% \\
4.2 \\
4.21 \\
4.29\end{array}$ & $\begin{array}{c}\text { mg. \% } \\
9.6 \\
9.7 \\
9.77\end{array}$ \\
\hline & $\begin{array}{l}\text { Abnormal serum dilution } \\
\text { (lymphosarcoma: } \\
\text { S. R., } \&, 38)\end{array}$ & $\begin{array}{l}1: 1 \\
1: 2 \\
1: 5\end{array}$ & $\begin{array}{l}296 \\
295.3 \\
296\end{array}$ & $\begin{array}{l}296 \\
295.5 \\
296\end{array}$ & $\begin{array}{l}34 \\
33.4 \\
33.6\end{array}$ & $\begin{array}{l}15.5 \\
15.25 \\
15.4\end{array}$ \\
\hline
\end{tabular}

TABLE III

Total mucoprotein (T.Mp.), mucoprotein-bound hexoses (Mh.) and hexosamine (Mha.) from serum treated with decreasing concentration of perchloric acid*

\begin{tabular}{|c|c|c|c|c|c|c|c|c|c|c|c|c|c|c|c|}
\hline \multirow[t]{2}{*}{$\begin{array}{l}\text { Molarities of } \\
\text { perchloric acid }\end{array}$} & \multicolumn{3}{|c|}{0.6} & \multicolumn{3}{|c|}{0.45} & \multicolumn{3}{|c|}{0.3} & \multicolumn{3}{|c|}{0.225} & \multicolumn{3}{|c|}{0.15} \\
\hline & T.Mp. & Mh. & Mha. & T.Mp. & Mh. & Mha. & T.Mp. & Mh. & Mha. & T.Mp. & Mh. & Mha. & T.Mp. & Mh. & Mha. \\
\hline Normal serum & $\begin{array}{c}\text { mg. \% } \\
98.8 \\
100\end{array}$ & $\begin{array}{c}m g . \% \\
6.2 \\
6.3\end{array}$ & $\begin{array}{l}m g . \% \\
11.5 \\
11.7\end{array}$ & $\begin{array}{c}\text { mg. \% } \\
98.6 \\
101\end{array}$ & $\begin{array}{c}\text { mg. } \% \\
6.2 \\
5.2\end{array}$ & $\begin{array}{l}m g . \% \\
11.5 \\
11.7\end{array}$ & $\begin{array}{c}m g . \% \\
98.8 \\
100\end{array}$ & $\begin{array}{c}\text { mg. } \% \\
6.1 \\
6.1\end{array}$ & $\begin{array}{c}m g . \% \\
11.6 \\
11.5\end{array}$ & $\begin{array}{c}\text { mg. \% } \\
98.8 \\
98\end{array}$ & $\begin{array}{c}m g . \% \\
6.1 \\
6.2\end{array}$ & $\begin{array}{l}\text { mg. \% } \\
11.5 \\
11.6\end{array}$ & $\begin{array}{c}\text { mg. } \% \\
98 \\
92\end{array}$ & $\begin{array}{c}m g . \% \\
5.75 \\
5.6\end{array}$ & $\begin{array}{c}m g . \% \\
11 \\
10.9\end{array}$ \\
\hline $\begin{array}{l}\text { Pathological } \\
\text { serum }\end{array}$ & $\begin{array}{l}190 \\
218\end{array}$ & $\begin{array}{l}12.6 \\
13.0\end{array}$ & $\begin{array}{l}15.1 \\
19.1\end{array}$ & $\begin{array}{l}190 \\
217\end{array}$ & $\begin{array}{l}12.5 \\
12.9\end{array}$ & $\begin{array}{l}15 \\
19.0\end{array}$ & $\begin{array}{l}190 \\
218 \\
\end{array}$ & $\begin{array}{l}12.6 \\
12.9\end{array}$ & $\begin{array}{l}15 \\
19.1\end{array}$ & $\begin{array}{l}189 \\
218 \\
\end{array}$ & $\begin{array}{l}12.6 \\
13.0\end{array}$ & $\begin{array}{l}15 \\
19.2\end{array}$ & $\begin{array}{l}162 \\
209\end{array}$ & $\begin{array}{l}12 \\
11.9 \\
\end{array}$ & $\begin{array}{l}14.5 \\
17.3 \\
\end{array}$ \\
\hline
\end{tabular}

* Averages of duplicate experiments on each serum.

stance, diagnosis was established by the usual hematologic criteria, and proven by either biopsy of involved tissues or autopsy findings. Since many diseases are accompanied by major alterations in content of serum glycoproteins and mucoproteins, cases selected for the present study, as far as could be determined, were free from infections, thrombosis, or hepatic and renal disorders. No definitive data appeared available to indicate whether treatment (myelo-suppressive drugs, X-ray radiation, and so on) may influence the level and composition of mucoproteins. ${ }^{7}$ In our experience, the amount and the composition of the serum mucoprotein fraction were more closely related to the state of remission or relapse, than to the effects of therapy. Usually, therefore, blood was collected from patients in clinical relapse, who were untreated at the time of blood collection.

\section{RESULTS}

The conclusions to follow are based primarily on the results of the $t$ test as a criterion of the statistical significance of our findings.

'Steroids, chemotherapeutic agents of various nature, $\mathrm{X}$-ray radiation, and so on might considerably modify the content and composition of mucoproteins in disease. The elevated content of mucopolysaccharide in the serum of patients with rheumatic fever decreases significantly following steroid therapy (9). In guinea pigs (10) and in rats (11), however, no correlation has been found between administration of steroids and variations in protein-bound hexoses.

\section{A. Normal sera (Table $V$ )}

Our findings agreed closely with those reported in the literature. They represented base values with which abnormal findings were compared.

TABLE IV

Sera studied in the course of these observations

Iron deficiency anemia

Pernicious anemia

Polycythemia vera

Myeloid metaplasia

Congenital spherocytosis

Acute leukemia : myelogenous histiocytic erythroblastic unclassified

Chronic leukemia : myelogenous lymphocytic

Hodgkin's disease : granuloma sarcoma

Lymphoblastoma

Lymphosarcoma

Reticulum cell sarcoma

Mycosis fungoides

Multiple myeloma

Acquired auto-immune hemolytic anemia

Idiopathic thrombocytopenic purpura

Lupus erythematosis disseminatus

Disseminated malignancies

Normal sera

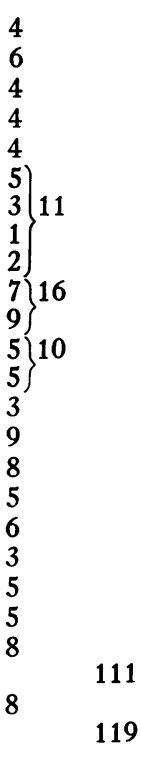


TABLE V

Serum protein-bound hexoses in normal subjects*

\begin{tabular}{|c|c|c|c|c|c|c|}
\hline Subject & T.P. & T.Ph. & T.Ha. & T.Mp. & Mh. & Mha. \\
\hline 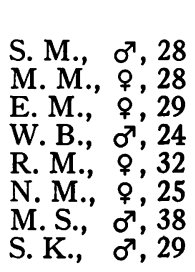 & $\begin{array}{c}m g . \% \\
7,600 \\
6,800 \\
7,000 \\
7,000 \\
7,200 \\
7,700 \\
7,500 \\
7,900\end{array}$ & $\begin{array}{c}m g . \% \\
100 \\
95 \\
125 \\
99 \\
120 \\
115 \\
107 \\
115\end{array}$ & $\begin{array}{c}m g . \% \\
100 \\
85 \\
107 \\
88 \\
100 \\
105 \\
98 \\
106\end{array}$ & $\begin{array}{c}m g . \% \\
95 \\
90 \\
105 \\
115 \\
85 \\
100 \\
90 \\
110\end{array}$ & $\begin{array}{r}\text { mg. \% } \\
5.355 \\
4.165 \\
8.500 \\
10.200 \\
4.250 \\
7.140 \\
4.505 \\
7.650\end{array}$ & $\begin{array}{r}m g . \% \\
10.20 \\
10.20 \\
12.75 \\
12.75 \\
9.35 \\
11.90 \\
9.35 \\
13.60\end{array}$ \\
\hline$\underset{\sigma}{\text { Average }}$ & $\begin{array}{r}7,337.5 \\
367.21\end{array}$ & $\begin{array}{c}109.5 \\
10.17\end{array}$ & $\begin{array}{l}98.6 \\
7.647\end{array}$ & $\begin{array}{c}98.8 \\
9.92\end{array}$ & $\begin{array}{l}6.471 \\
2.098\end{array}$ & $\begin{array}{r}11.26 \\
1.58\end{array}$ \\
\hline
\end{tabular}

* As in each table, analytical values and standard deviation $(\sigma)$ are given for the following: Total protein (T.P.), protein-bound hexoses (T.Ph.), total hexosamine (T.Ha), total mucoprotein (T.Mp.), mucoprotein-bound hexoses (Mh.), and mucoprotein-bound hexosamine (Mha).

TABLE VI

Serum protein-bound hexoses in acute leukemia

\begin{tabular}{|c|c|c|c|c|c|c|c|}
\hline Type & Case & T.P. & T.Ph. & T.Ha. & T.Mp. & Mh. & Mha. \\
\hline $\begin{array}{l}\text { Unclassified } \\
\text { Granulocytic } \\
\text { Granulocytic } \\
\text { Granulocytic } \\
\text { Histiocytic } \\
\text { Granulocytic } \\
\text { Histiocytic } \\
\text { Erythroblastic } \\
\text { Lymphocytic } \\
\text { Granulocytic } \\
\text { Granulocytic }\end{array}$ & $\begin{array}{l}\text { No. } 3005 \\
\text { No. } 11 \\
\text { No. } 12 \\
\text { No. } 13 \\
\text { D. M., o, } 38 \\
\text { L. S., \&, } 76 \\
\text { C. P., ơ, } 29 \\
\text { P. McG., ơ, } 52 \\
\text { J. T., ơ, } 5 \\
\text { C. V., \&, } 54 \\
\text { R. Q., ơ, } 57\end{array}$ & $\begin{array}{l}m g . \% \\
7,300 \\
7,600 \\
7,800 \\
7,600 \\
7,200 \\
7,200 \\
7,300 \\
7,000 \\
6,100 \\
7,000 \\
6,100\end{array}$ & $\begin{array}{l}m g . \% \\
138 \\
130 \\
135 \\
160 \\
147 \\
147 \\
150 \\
160 \\
190 \\
190 \\
185\end{array}$ & $\begin{array}{l}m g . \% \\
128 \\
115 \\
120 \\
150 \\
140 \\
120 \\
120 \\
140 \\
120 \\
140 \\
110\end{array}$ & $\begin{array}{l}\text { mg. \% } \\
186 \\
246 \\
171 \\
228 \\
310 \\
253 \\
103 \\
123 \\
123 \\
140 \\
117\end{array}$ & $\begin{array}{r}m g . \% \\
8.84 \\
27.63 \\
24.40 \\
12.50 \\
42.50 \\
38.25 \\
15.30 \\
20.91 \\
8.67 \\
16.58 \\
9.69\end{array}$ & $\begin{array}{l}m g . \% \\
13.60 \\
23.80 \\
21.25 \\
12.75 \\
12.75 \\
10.20 \\
13.60 \\
18.70 \\
10.20 \\
15.30 \\
11.90\end{array}$ \\
\hline $\begin{array}{c}\text { Average } \\
\sigma \\
t\end{array}$ & & $\begin{array}{r}7,109.1 \\
531.64 \\
0.989\end{array}$ & $\begin{array}{r}157.5 \\
19.88 \\
5.63\end{array}$ & $\begin{array}{r}127.5 \\
12.3 \\
4.95\end{array}$ & $\begin{array}{r}181.8 \\
65.34 \\
3.28\end{array}$ & $\begin{array}{r}20.48 \\
11.14 \\
4.38\end{array}$ & $\begin{array}{r}14.91 \\
4.26 \\
2.15\end{array}$ \\
\hline
\end{tabular}

TABLE VII

Serum protein-bound hexoses in chronic leukemia (myelogenous and lymphocytic)

\begin{tabular}{|c|c|c|c|c|c|c|c|}
\hline Case & Type & T.P. & T.Ph. & T.Ha. & T.Mp. & Mh. & Mha. \\
\hline 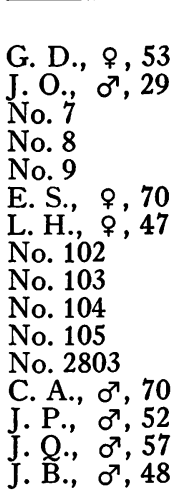 & $\begin{array}{l}\text { Granulocytic } \\
\text { Granulocytic } \\
\text { Granulocytic } \\
\text { Granulocytic } \\
\text { Granulocytic } \\
\text { Granulocytic } \\
\text { Granulocytic } \\
\text { Lymphocytic } \\
\text { Lymphocytic } \\
\text { Lymphocytic } \\
\text { Lymphocytic } \\
\text { Lymphocytic } \\
\text { Lymphocytic } \\
\text { Lymphocytic } \\
\text { Lymphocytic } \\
\text { Lymphocytic }\end{array}$ & $\begin{array}{l}m g . \% \\
5,900 \\
6,900 \\
6,000 \\
6,000 \\
6,500 \\
7,300 \\
7,000 \\
7,600 \\
7,200 \\
6,300 \\
5,800 \\
7,700 \\
6,400 \\
5,800 \\
7,400 \\
6,700\end{array}$ & $\begin{array}{l}m g . \% \\
125 \\
130 \\
85 \\
175 \\
135 \\
152 \\
130 \\
125 \\
140 \\
135 \\
145 \\
130 \\
135 \\
115 \\
140 \\
134\end{array}$ & $\begin{array}{l}m g . \% \\
110 \\
115 \\
120 \\
145 \\
118 \\
120 \\
110 \\
115 \\
150 \\
130 \\
170 \\
105 \\
95 \\
128 \\
150 \\
160\end{array}$ & $\begin{array}{l}m g . \% \\
113 \\
118.6 \\
105.2 \\
155 \\
165 \\
145 \\
130 \\
128 \\
140 \\
140 \\
228 \\
100 \\
92 \\
100 \\
134 \\
195\end{array}$ & $\begin{array}{r}m g . \% \\
12.50 \\
15.30 \\
15.56 \\
9.78 \\
10.29 \\
9.35 \\
8.16 \\
17.00 \\
13.69 \\
18.28 \\
32.90 \\
11.22 \\
11.05 \\
10.20 \\
10.12 \\
15.30\end{array}$ & $\begin{array}{l}m g . \% \\
13.60 \\
17.00 \\
14.45 \\
12.75 \\
11.05 \\
10.20 \\
11.90 \\
18.70 \\
15.30 \\
17.00 \\
16.15 \\
10.20 \\
8.50 \\
11.90 \\
11.05 \\
14.45\end{array}$ \\
\hline $\begin{array}{c}\text { Average } \\
\sigma \\
t\end{array}$ & & $\begin{array}{r}6,656.3 \\
639.31 \\
2.67\end{array}$ & $\begin{array}{r}133.2 \\
17.96 \\
3.26\end{array}$ & $\begin{array}{r}127.6 \\
20.73 \\
3.68\end{array}$ & $\begin{array}{c}136.8 \\
35.05 \\
2.8\end{array}$ & $\begin{array}{c}13.79 \\
5.73 \\
3.2\end{array}$ & $\begin{array}{c}13.39 \\
2.827 \\
1.88\end{array}$ \\
\hline
\end{tabular}


HUMAN MUCOPROTEIN FRACTION IN HEMATOLOGIC DISEASES

TABLE VIII

Serum protein-bound hexoses in Hodgkin's disease

\begin{tabular}{|c|c|c|c|c|c|c|c|}
\hline C.ase & Type & T.P. & T.Ph. & T.Ha. & $\mathrm{T} . \mathrm{Mp}$ & Mh. & Mha. \\
\hline 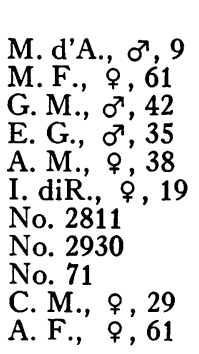 & $\begin{array}{l}\text { Sarcoma } \\
\text { Sarcoma } \\
\text { Sarcoma } \\
\text { Sarcoma } \\
\text { Sarcoma } \\
\text { Sarcoma } \\
\text { Granuloma } \\
\text { Granuloma } \\
\text { Granuloma } \\
\text { Granuloma } \\
\text { Granuloma }\end{array}$ & $\begin{array}{l}m g . \% \\
6,700 \\
6,200 \\
6,500 \\
7,700 \\
6,700 \\
7,300 \\
7,800 \\
7,000 \\
6,800 \\
6,500 \\
7,600\end{array}$ & $\begin{array}{l}m g . \% \\
230 \\
158 \\
190 \\
200 \\
200 \\
221 \\
92 \\
215 \\
198 \\
225 \\
153\end{array}$ & $\begin{array}{l}m g . \% \\
170 \\
95 \\
98 \\
175 \\
145 \\
155 \\
100 \\
140 \\
160 \\
182 \\
130\end{array}$ & $\begin{array}{l}m g . \% \\
190 \\
158 \\
100 \\
204 \\
210 \\
190 \\
103 \\
213 \\
175 \\
230 \\
153\end{array}$ & $\begin{array}{r}m g . \% \\
32.30 \\
9.18 \\
8.67 \\
7.74 \\
22.95 \\
15.73 \\
11.31 \\
11.90 \\
13.60 \\
26.35 \\
5.19\end{array}$ & $\begin{array}{l}m g . \% \\
34.00 \\
15.30 \\
14.45 \\
13.60 \\
15.30 \\
9.35 \\
12.75 \\
12.75 \\
11.90 \\
16.15 \\
12.75\end{array}$ \\
\hline $\begin{array}{c}\text { Average } \\
\sigma \\
t\end{array}$ & & $\begin{array}{r}6,981.8 \\
516.67 \\
1.76\end{array}$ & $\begin{array}{r}189.3 \\
38.92 \\
5.48\end{array}$ & $\begin{array}{r}141 \\
30.18 \\
3.65\end{array}$ & $\begin{array}{r}175 \\
43.66 \\
4.81\end{array}$ & $\begin{array}{c}14.99 \\
8.201 \\
2.73\end{array}$ & $\begin{array}{c}15.3 \\
6.183 \\
1.72\end{array}$ \\
\hline
\end{tabular}

\section{B. Acute leukemia (Table VI)}

There was a statistically significant rise of total hexosamine, total mucoprotein and hexoses bound to protein and mucoprotein fractions.

\section{Chronic leukemias (Table VII)}

The findings, essentially independent of the histologic type of the disease, were similar to those observed in the acute leukemias, although quantitatively less significant.

\section{Hodgkin's disease (Table VIII)}

There was a substantial rise in total hexosamine, total mucoprotein and protein-bound hexoses.
The rise in mucoprotein-bound hexoses seemed statistically less significant. No appreciable difference was observed between the granuloma and the sarcoma type of the disease.

\section{E. Lymphosarcoma (Table IX)}

Total hexosamine, total mucoprotein and mucoprotein-bound hexoses were significantly increased. There was, in fact, a rather spectacular rise in total mucoprotein and mucoprotein-bound hexoses, which seemed typical of the disease. There was no significant difference in findings between patients in this group who had been classified by the pathologist as suffering from either lymphosarcoma or lymphoblastoma.

TABLE IX

Serum protein-bound hexoses in lymphosarcoma

\begin{tabular}{|c|c|c|c|c|c|c|}
\hline Case & T.P. & T.Ph. & T.Ha. & T.Mp. & Mh. & Mha. \\
\hline 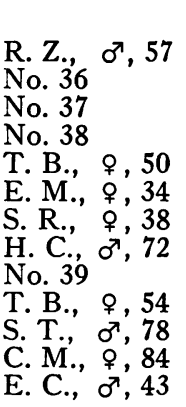 & $\begin{array}{c}m g . \% \\
6,100 \\
5,000 \\
7,500 \\
6,100 \\
7,700 \\
7,200 \\
7,800 \\
7,000 \\
7,200 \\
5,800 \\
5,600 \\
7,600 \\
6,300\end{array}$ & $\begin{array}{l}m g . \% \\
207 \\
190 \\
203 \\
135 \\
190 \\
230 \\
255 \\
218 \\
203 \\
290 \\
240 \\
195 \\
280\end{array}$ & $\begin{array}{l}m g . \% \\
150 \\
140 \\
180 \\
100 \\
158 \\
170 \\
160 \\
125 \\
120 \\
120 \\
170 \\
140 \\
120\end{array}$ & $\begin{array}{l}m g . \% \\
211 \\
190 \\
301.2 \\
115 \\
200 \\
239 \\
295 \\
179 \\
195 \\
197 \\
279 \\
242 \\
181\end{array}$ & $\begin{array}{l}\text { mg. \% } \\
34.27 \\
51.00 \\
46.75 \\
10.88 \\
32.05 \\
38.93 \\
33.92 \\
19.13 \\
38.68 \\
29.75 \\
34.00 \\
42.50 \\
31.88\end{array}$ & $\begin{array}{r}m g . \% \\
11.90 \\
17.85 \\
18.70 \\
9.35 \\
11.90 \\
13.60 \\
15.30 \\
11.90 \\
11.90 \\
11.90 \\
13.60 \\
13.60 \\
14.45\end{array}$ \\
\hline $\begin{array}{c}\text { Average } \\
\sigma \\
t\end{array}$ & $\begin{array}{r}6,684.6 \\
878.67 \\
1.97\end{array}$ & $\begin{array}{r}218.2 \\
32.74 \\
2.33\end{array}$ & $\begin{array}{c}142.5 \\
23.48 \\
4.9\end{array}$ & $\begin{array}{r}217.2 \\
50.42 \\
6.16\end{array}$ & $\begin{array}{r}34.13 \\
10.19 \\
7.07\end{array}$ & $\begin{array}{r}13.53 \\
2.48 \\
2.19\end{array}$ \\
\hline
\end{tabular}


TABLE $\mathrm{X}$

Serum protein-bound hexoses in reticulum-cell sarcoma

\begin{tabular}{|c|c|c|c|c|c|c|}
\hline Case & T.P. & T.Ph. & T.Ha. & T.Mp. & Mh. & Mha. \\
\hline $\begin{array}{l}\text { C. B., o, } 57 \\
\text { W. B., ơ, } 80 \\
\text { J. M., ơ, } 51 \\
\text { No. } 34 \\
\text { M. C., ơ, } 67\end{array}$ & $\begin{array}{c}m g . \% \\
6,800 \\
5,600 \\
6,000 \\
7,600 \\
6,500\end{array}$ & $\begin{array}{l}m g . \% \\
245 \\
205 \\
210 \\
275 \\
180\end{array}$ & $\begin{array}{l}m g . \% \\
170 \\
140 \\
165 \\
130 \\
110\end{array}$ & $\begin{array}{c}m g . \% \\
394.0 \\
160.7 \\
217.0 \\
153.0 \\
195.0\end{array}$ & $\begin{array}{r}\text { mg. \% } \\
38.25 \\
7.74 \\
7.40 \\
32.47 \\
10.80\end{array}$ & $\begin{array}{l}m g . \% \\
13.60 \\
13.60 \\
16.15 \\
12.75 \\
11.05\end{array}$ \\
\hline $\begin{array}{c}\text { Average } \\
\sigma \\
t\end{array}$ & $\begin{array}{r}6,500 \\
687.02 \\
2.62\end{array}$ & $\begin{array}{r}223 \\
33.24 \\
\quad 8.17\end{array}$ & $\begin{array}{c}143 \\
22.27 \\
4.7\end{array}$ & $\begin{array}{r}223.9 \\
88.18 \\
3.62\end{array}$ & $\begin{array}{r}19.33 \\
13.27 \\
2.51\end{array}$ & $\begin{array}{c}13.43 \\
1.648 \\
2.18\end{array}$ \\
\hline
\end{tabular}

TABLE XI

Serum protein-bound hexoses in mycosis fungoides

\begin{tabular}{|c|c|c|c|c|c|c|}
\hline Case & T.P. & T.Ph. & T.Ha. & T.Mp. & Mh. & Mha. \\
\hline $\begin{array}{l}\text { T. C. } \\
\text { T. H. } \\
\text { P. C. } \\
\text { S. A. } \\
\text { B. B. }\end{array}$ & $\begin{array}{l}\text { mg. \% } \\
7,600 \\
7,200 \\
4,400 \\
5,600 \\
6,300\end{array}$ & $\begin{array}{l}m g . \% \\
140 \\
135 \\
135 \\
130 \\
139\end{array}$ & $\begin{array}{l}m g . \% \\
125 \\
110 \\
120 \\
160 \\
204\end{array}$ & $\begin{array}{l}m g . \% \\
273 \\
183 \\
127 \\
137 \\
180\end{array}$ & $\begin{array}{c}m g . \% \\
25.245 \\
21.505 \\
24.820 \\
16.745 \\
22.270\end{array}$ & $\begin{array}{r}m g . \% \\
11.05 \\
8.50 \\
9.35 \\
6.80 \\
10.20\end{array}$ \\
\hline $\begin{array}{c}\text { Average } \\
\sigma \\
\mathrm{t}\end{array}$ & $\begin{array}{r}6,220 \\
1,146.12 \\
2.34\end{array}$ & $\begin{array}{r}135.8 \\
3.54 \\
4.74\end{array}$ & $\begin{array}{r}143.8 \\
34.46 \\
3.28\end{array}$ & $\begin{array}{r}180 \\
51.62 \\
3.97\end{array}$ & $\begin{array}{c}22.117 \\
3.044 \\
9.46\end{array}$ & $\begin{array}{l}9.18 \\
1.462 \\
2.2\end{array}$ \\
\hline
\end{tabular}

TABLE XII

Serum protein-bound hexoses in lupus erythematosus disseminatus

\begin{tabular}{|c|c|c|c|c|c|c|}
\hline Case & T.P. & T.Ph. & T.Ha. & T.Mp. & $\mathrm{Mh}$. & Mha. \\
\hline $\begin{array}{l}\text { E. M., ơ } 78 \\
\text { H. McC., } \$, 33 \\
\text { No. } 51 \\
\text { Sr. E., \&, } 54 \\
\text { S. O'H., \&, } 73\end{array}$ & $\begin{array}{l}m g . \% \\
7,200 \\
6,200 \\
7,400 \\
7,600 \\
7,100\end{array}$ & $\begin{array}{l}m g . \% \\
120 \\
107 \\
150 \\
140 \\
168.4\end{array}$ & $\begin{array}{c}m g . \% \\
150 \\
135 \\
90 \\
140 \\
155.0\end{array}$ & $\begin{array}{r}m g . \% \\
195.0 \\
68.9 \\
190.0 \\
123.0 \\
166.0\end{array}$ & $\begin{array}{c}m g . \% \\
23.375 \\
6.96 \\
34.00 \\
9.18 \\
11.64\end{array}$ & $\begin{array}{c}m g . \% \\
11.90 \\
11.05 \\
13.60 \\
11.05 \\
12.75\end{array}$ \\
\hline $\begin{array}{c}\text { Average } \\
\sigma \\
\mathrm{t}\end{array}$ & $\begin{array}{r}7,100 \\
481.66 \\
0.92\end{array}$ & $\begin{array}{r}137.1 \\
21.69 \\
2.77\end{array}$ & $\begin{array}{c}134 \\
23.108 \\
3.68\end{array}$ & $\begin{array}{r}148.6 \\
46.91 \\
2.53\end{array}$ & $\begin{array}{c}17.03 \\
10.201 \\
2.68\end{array}$ & $\begin{array}{l}12.07 \\
0.9913 \\
0.93\end{array}$ \\
\hline
\end{tabular}

TABLE XIII

Serum protein-bound hexoses in multiple myeloma

\begin{tabular}{|c|c|c|c|c|c|c|c|}
\hline Case & Type & T.P. & T.Ph. & T.Ha. & T.Mp. & Mh. & Mha. \\
\hline $\begin{array}{l}\text { G. G., } \sigma^{7}, 57 \\
\text { No. } 21 \\
\text { N. B., } \$, 62 \\
\text { J. C., ơ, } 59 \\
\text { D. S., ơ, } 72 \\
\text { No. } 22\end{array}$ & $\begin{array}{l}\text { Plasmocytic leukemia } \\
\beta \text {-myeloma } \\
\beta \text {-myeloma } \\
\gamma \text {-myeloma } \\
\gamma \text {-myeloma } \\
\gamma \text {-myeloma }\end{array}$ & $\begin{array}{r}m g . \% \\
10,000 \\
9,500 \\
10,800 \\
9,100 \\
10,200 \\
9,000\end{array}$ & $\begin{array}{l}m g . \% \\
400 \\
200 \\
300 \\
180 \\
200 \\
250\end{array}$ & $\begin{array}{l}\text { mg. \% } \\
170 \\
160 \\
180 \\
160 \\
150 \\
170\end{array}$ & $\begin{array}{c}m g . \% \\
91 \\
217 \\
242 \\
117 \\
131 \\
190\end{array}$ & $\begin{array}{c}m g . \% \\
3.57 \\
22.27 \\
11.15 \\
11.05 \\
19.55 \\
17.00\end{array}$ & $\begin{array}{l}m g . \% \\
18.70 \\
20.40 \\
16.15 \\
17.00 \\
17.00 \\
15.30\end{array}$ \\
\hline $\begin{array}{c}\text { Average } \\
\sigma \\
t\end{array}$ & & $\begin{array}{c}9,766.6 \\
634.21 \\
7.6\end{array}$ & $\begin{array}{l}255 \\
76.103 \\
4.51\end{array}$ & $\begin{array}{c}165 \\
9.574 \\
12.16\end{array}$ & $\begin{array}{r}165 \\
55.06 \\
2.81\end{array}$ & $\begin{array}{c}14.1 \\
6.242 \\
2.72\end{array}$ & $\begin{array}{r}17.43 \\
1.68 \\
5.95\end{array}$ \\
\hline
\end{tabular}


HUMAN MUCOPROTEIN FRACTION IN HEMATOLOGIC DISEASES

TABLE XIV

Serum protein-bound hexoses in idiopathic thrombocytopenic purpura

\begin{tabular}{|c|c|c|c|c|c|c|c|}
\hline Case & Type & T.P. & T.Ph. & T.Ha. & T.Mp. & Mh. & Mha. \\
\hline 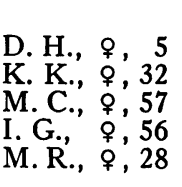 & $\begin{array}{l}\text { Acute } \\
\text { Chronic } \\
\text { Acute } \\
\text { Chronic } \\
\text { Chronic }\end{array}$ & $\begin{array}{c}\text { mg. \% } \\
7,600 \\
6,500 \\
7,800 \\
7,200 \\
7,500\end{array}$ & $\begin{array}{l}\text { mg. \% } \\
180 \\
185 \\
218 \\
145 \\
175\end{array}$ & $\begin{array}{l}m g . \% \\
125 \\
160 \\
150 \\
130 \\
150\end{array}$ & $\begin{array}{l}m g . \% \\
92 \\
100 \\
140 \\
125 \\
145\end{array}$ & $\begin{array}{l}m g . \% \\
10.6 \\
12 \\
11.2 \\
9.6 \\
8.9\end{array}$ & $\begin{array}{l}m g . \% \\
12 \\
15 \\
14 \\
16 \\
15\end{array}$ \\
\hline $\begin{array}{c}\text { Average } \\
\sigma \\
t\end{array}$ & & $\begin{array}{r}7,320 \\
453.43 \\
0.07\end{array}$ & $\begin{array}{r}180.6 \\
23.31 \\
6.79\end{array}$ & $\begin{array}{r}143 \\
13.27 \\
6.83\end{array}$ & $\begin{array}{r}120.4 \\
21.13 \\
2.29\end{array}$ & $\begin{array}{c}10.46 \\
1.105 \\
3.61\end{array}$ & $\begin{array}{c}14.4 \\
1.357 \\
3.39\end{array}$ \\
\hline
\end{tabular}

\section{F. Reticulum-cell sarcoma (Table X)}

Patients included in this group showed considerable variations in histologic characteristics and clinical course, but equal variability of chemical findings. The statistical analysis revealed significant rise in total hexosamine and protein-bound hexoses only.

\section{G. Mycosis fungoides (Table XI)}

There was a significant rise in hexoses bound to both the protein and the mucoprotein fraction, although the increase in total mucoprotein was equivocal. The rise in total hexosamine indicated by the value of $\sigma$ was apparently of no real significance according to the evaluation of the $t$ test.

\section{H. Lupus erythematosus (Table XII)}

There was a superficial resemblance of the findings to those in mycosis fungoides. Statistically, however, the only significant variation was an equivocal rise of the total hexosamine.

\section{Multiple myeloma (Table XIII)}

Serum of these patients showed a typical pattern. There was a significant rise in total protein, protein-bound hexoses, total and mucoproteinbound hexosamine.

\section{J. Idiopathic thrombocytopenic purpura (Table $X I V)$}

Total hexosamine and protein-bound hexoses were significantly elevated. The rise in total mucoprotein was equivocal.

\section{$K$. Disseminated malignancies (Table $X V$ )}

Although the sera studied in this group were obtained from a heterogenous group of patients with regard to site of the primary lesion, findings were uniform. There was significant decrease

TABLE XV

Serum protein-bound hexoses in generalized malignancies

\begin{tabular}{|c|c|c|c|c|c|c|c|}
\hline Case & Type & T.P. & T.Ph. & T.Ha. & T.Mp. & Mh. & Mha. \\
\hline $\begin{array}{l}\text { R. R., ơ, } 62 \\
\text { R. P., } \$, 52\end{array}$ & $\begin{array}{l}\text { Melanosarcoma } \\
\text { Adenoacanthoma } \\
\text { of ovary }\end{array}$ & $\begin{array}{l}m g . \% \\
6,000 \\
6,900\end{array}$ & $\begin{array}{l}m g . \% \\
180 \\
190\end{array}$ & $\begin{array}{l}m g . \% \\
130 \\
135\end{array}$ & $\begin{array}{l}m g . \% \\
210.6 \\
215\end{array}$ & $\begin{array}{l}m g . \% \\
11.5 \\
10.2\end{array}$ & $\begin{array}{l}m g . \% \\
14.0 \\
13.0\end{array}$ \\
\hline H. H., $\$, 60$ & $\begin{array}{l}\text { Adenocarcinoma } \\
\text { of breast }\end{array}$ & 6,300 & 185 & 130 & 220 & 12.5 & 14.5 \\
\hline J. N., ఠ, 60 & $\begin{array}{l}\text { Carcinoma of } \\
\text { prostate }\end{array}$ & 5,900 & 170 & 125 & 177 & 11.3 & 13.2 \\
\hline H. H., ơ , 36 & $\begin{array}{l}\text { Anaplastic } \\
\text { carcinoma }\end{array}$ & 5,200 & 175 & 138 & 196 & 12.2 & 14.4 \\
\hline H. N., ơ , 55 & $\begin{array}{l}\text { Carcinoma of } \\
\text { rectum }\end{array}$ & 6,700 & 164 & 125 & 173 & 9.1 & 13.0 \\
\hline L. S., $\quad$ ค, 38 & $\begin{array}{l}\text { Carcinoma of } \\
\text { stomach }\end{array}$ & 4,500 & 167 & 130 & 180 & 10.0 & 13.8 \\
\hline N. H., ㅇ, 67 & $\begin{array}{l}\text { Carcinoma of } \\
\text { stomach }\end{array}$ & 7,100 & 185 & 142 & 209 & 12.7 & 14.6 \\
\hline $\begin{array}{c}\text { Average } \\
\sigma \\
t\end{array}$ & & $\begin{array}{r}6,075 \\
825.76 \\
4.98\end{array}$ & $\begin{array}{r}177 \\
8.86 \\
11.94\end{array}$ & $\begin{array}{c}131.9 \\
5.644 \\
9.38\end{array}$ & $\begin{array}{l}197.6 \\
17.48 \\
14.31\end{array}$ & $\begin{array}{l}11.2 \\
1.219 \\
6.84\end{array}$ & $\begin{array}{l}13.8 \\
0.629 \\
3.92\end{array}$ \\
\hline
\end{tabular}


in total proteins, with significant elevation of all other serum constituents. Total hexosamine seemed relatively more increased than hexosamine bound to the mucoprotein fraction.

\section{Miscellaneous group}

Our findings were normal in 25 patients with such conditions as polycythemia vera (four), acquired idiopathic auto-immune hemolytic anemia without evidence of collagen disease or malignancy (three), congenital spherocytosis (four), pernicious anemia (six), iron deficiency anemia (four) and myeloid metaplasia (four).

\section{DISCUSSION}

The significance of quantitative changes of the serum mucoprotein fraction in disease has been fully reviewed by Greenspan (12). This author concluded that the total mucoprotein fraction of serum is increased in trauma and in diseases accompanied by inflammatory, neoplastic or degenerative tissue changes, while it is decreased in parenchymal hepatic disease, endocrine dysfunctions and nephrosis. Only fragmentary work, however, seems to have been done on the qualitative composition of the serum mucoprotein fraction in disease. This paper attempts to correlate changes in the mucoprotein fraction of serum in patients with several hematologic diseases as compared with normal sera. This has been done through the study of quantitative variations of some constituents of the serum mucoprotein fraction and of their comparative distribution between the protein and mucoprotein fractions.

Figure 1 and Table XVI are a summary of our work. In Figure 1, the height of columns is based on the calculated averages. With few exceptions, these variations appear significant, as indicated by the results of the $t$ test whose evaluation is offered in Table XVI and is the base for the discussion to follow. The decrease of total protein, noted in most conditions studied on the basis of average figures, was significant only in disseminated malignancies. Absolute rise of serum mucoprotein seemed a common denominator of most conditions studied although significant only in neoplastic processes (acute leukemias, Hodgkin's disease, lymphosarcoma and disseminated malignancies). An increase in protein-bound hexoses was again noted in practically all conditions studied and was statistically significant in the majority of them (acute and chronic leukemias, Hodgkin's disease, reticulum cell sarcomas, mycosis fungoides, multiple myeloma, idiopathic thrombocytopenic purpura and disseminated malignancies). In some conditions (acute and chronic leukemias, mycosis fungoides, idiopathic thrombocytopenic purpura, disseminated malignancies) there was significant increase in mucoprotein-bound hexoses while interesting dissociations were also observed. Thus in Hodgkin's disease and in multiple myeloma only the rise in protein-bound hexoses was significant; in lymphosarcoma, only the rise of mucoprotein-bound hexoses. In mycosis fungoides the rise in mucoprotein-bound hexoses was much more significant than that of the hexoses bound to protein. The opposite situation held true for idiopathic thrombocytopenic purpura. Total hexosamine was also significantly increased in acute and chronic leukemias, Hodgkin's disease, lymphosarcoma, reticulum cell sarcoma, multiple myeloma, idiopathic thrombocytopenic purpura

TABLE XVI

Statistical evaluation of results by the $t$ test: $A$ summary*

\begin{tabular}{|c|c|c|c|c|c|c|}
\hline & T.P. & T.Ph. & T.Ha. & T.Mp. & Mh. & Mha. \\
\hline Acute leukemias & - & + & + & + & + & - \\
\hline Chronic leukemias & \pm & + & + & \pm & + & - \\
\hline Hodgkin's disease & $\overline{-}$ & + & + & + & - & - \\
\hline Lymphosarcoma & - & - & $\dot{t}$ & + & ++ & - \\
\hline Reticulum cell sarcoma & - & + & + & - & - & - \\
\hline Mycosis fungoides & - & + & - & \pm & $+t$ & - \\
\hline Lupus erythematosus & - & - & \pm & $\overline{-}$ & - & - \\
\hline Multiple myeloma & + & + & ++ & - & - & + \\
\hline Idiopathic thrombocytopenic purpura & - & + & + & - & \pm & - \\
\hline Generalized malignancies & $+(\downarrow)$ & ++ & ++ & ++ & + & + \\
\hline
\end{tabular}

$*-$ insignificant; \pm , equivocal; + , significant; and ++ , very significant. Variations represented increase from normal with the exception of the group "generalized malignancies," where values for T.P. were lower than normal. 


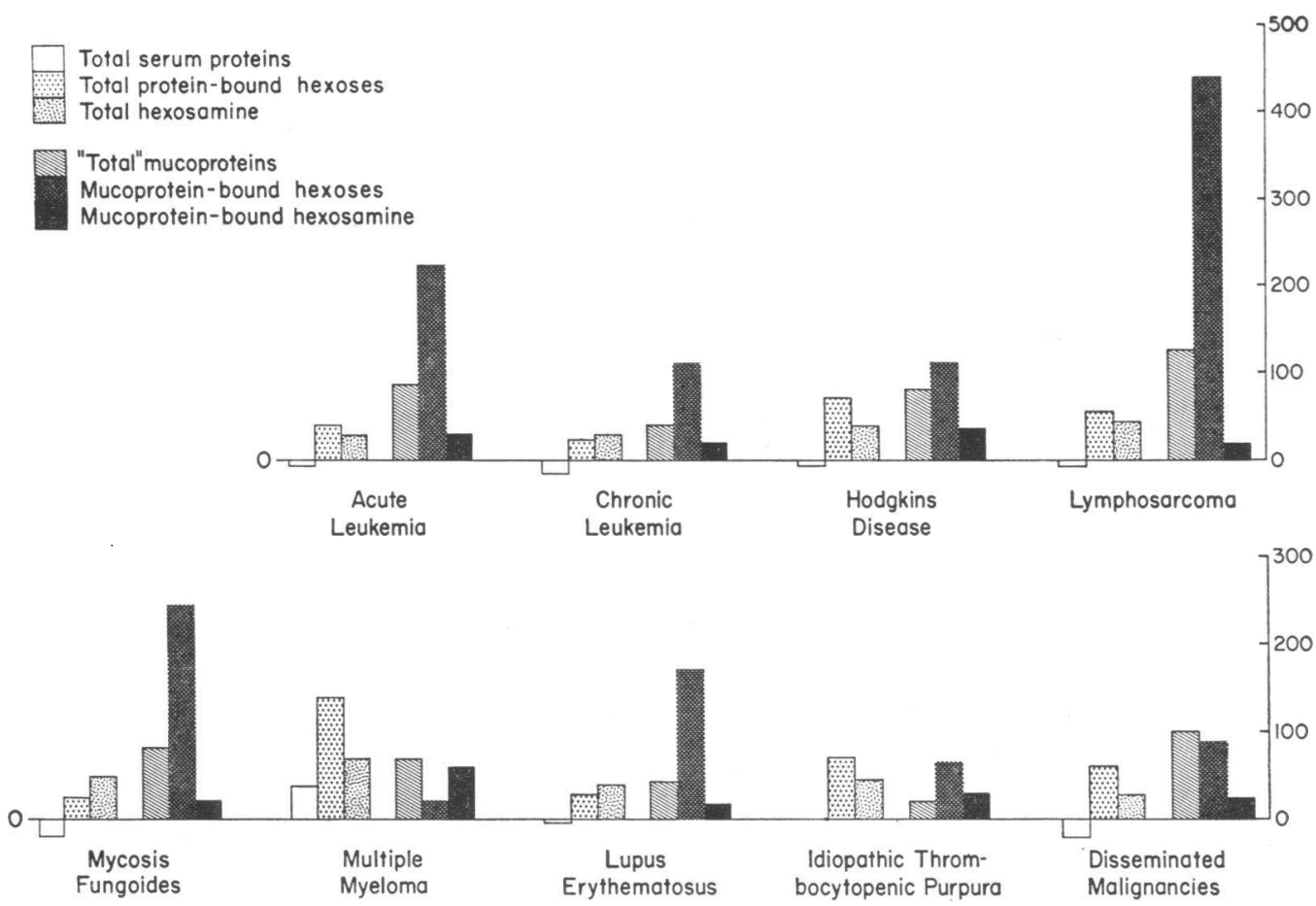

Fig. 1. Composition of Mucoproteins in Various Hematologic Disorders-A Summary

Figures represent percentage variations from average normal values, indicated by the horizontal line. Also given for comparison are variations in serum total proteins, total protein-bound hexoses and total hexosamine.

and disseminated malignancies; rise was equivocal in lupus erythematosus. It was of interest that mucoprotein-bound hexosamine was signifcantly elevated only in multiple myeloma and in disseminated malignancies. In a few instances, the combined variations of the serum constituents produced a distinctive pattern, as in multiple myeloma, lymphosarcoma, and disseminated malignancies. The potential diagnostic value of the changes observed, however, should perhaps be minimized, as these occurred only in patients in fairly advanced stages of the disease.

Also of interest were the findings indicating that the distribution of hexoses between the protein and mucoprotein fractions and the ratio of total hexosamine to mucoprotein-bound hexosamine were much different in normal than in many pathologic conditions. These findings raised the necessary question as to whether the composition of the serum mucoprotein fraction may be different in health as compared to disease and, perhaps, even from one disease state to another. Unpublished electrophoretic studies in this Laboratory have given further support to this possibility, showing an overall anomaly of electrophoretic migration of mucoprotein in disease. Thus, its constituents are usually recovered from the $\alpha_{1}-, \alpha_{2}$, and $\beta$-fractions; in pathologic conditions they may be recovered from the $\gamma$-fraction. Other supportive evidence can be obtained from the literature which suggests the largest amount of protein-bound hexoses to be found with the $\alpha$-globulins in most disease states (13-15). In carcinoma and in rheumatic fever, however, protein-bound hexoses are most abundant in the albumin fraction $(16,17)$. Bollett $(18)$ has recently confirmed an increase of hexosamine in the mucoprotein fraction of serum in a variety of illnesses, obtaining results which are essentially similar to ours when the same disease states were studied. $\mathrm{He}$ found also that such increase was limited to the hexosamine recovered from the $\alpha_{1}$ paper electrophoretic fraction. Finally, there is additional evidence, although fragmentary, of a chemical nature. An increase in the ratio hexose: tyrosine in the mucoprotein fraction has been reported in 
rheumatoid arthritis, gout (15) and experimental scurvy; it is not found, however, in many other conditions where this ratio has been studied (19, 20).

All these data seem to support the hypothesis that the mucoprotein fraction of serum in disease may be of abnormal composition. It should be remembered, however, that this fraction is of considerable heterogeneity and is possibly nothing more than a mosaic, combining several units. A single increase in content of one of the various fractions might well result in a simulated abnormal structure for the entire mucoprotein.

\section{SUMMARY}

1. Total protein, total protein-bound hexoses, total hexosamine, total mucoprotein, mucoproteinbound hexoses and hexosamine were studied in a series of 111 patients with various hematologic disorders and in 8 healthy individuals.

2. The mucoprotein fraction and total hexosamine were quantitatively increased in diseases of a neoplastic nature. Significant variations of the protein-bound and mucoprotein-bound hexoses were observed in many instances. Fairly distinctive patterns were found in a few diseases (multiple myeloma, lymphosarcoma, and disseminated malignancies).

3. The various anomalies observed suggested that the composition of the mucoprotein fraction of serum may be abnormal in disease.

\section{ACKNOWLEDGMENTS}

Dr. Margarida N. de Magalhaes performed preliminary determinations of total mucoprotein. Mr. Leo Munich assisted in the statistical evaluation of data.

\section{REFERENCES}

1. Meyer, K., Mucoids and glycoproteins in Advances in Protein Chemistry, M. L. Anson and J. T. Edsall, Eds. New York, Academic Press Inc., 1945, vol. 2, page 249.

2. Winzler, R. L., Devor, A. W., Mehl, J. W., and Smyth, I. M., Studies on the mucoproteins of human plasma. I. Determination and isolation. J. Clin. Invest., 1948, 27, 609.

3. Rimington, C., Seromucoid and the bound carbohydrate of the serum proteins. Biochem. J., 1940, 34, 931.

4. Hewitt, L. F., Separation of serum albumin into two fractions. II. Observations on the nature of the glycoprotein fraction. Biochem. J., 1937, 31, 360.
5. Gornall, A. G., Bardawill, C. J., and David, M. M., Determination of serum proteins by means of biuret reaction. J. Biol. Chem., 1949, 177, 751.

6. Sheppard, F., and Everett, M. R., Determination of carbohydrates in proteins (abstract). J. Biol. Chem., 1937, 119, 1xxxix.

7. Blair, M. M., Elementary Statistics with General Applications. New York, H. Holt and Co., 1949, page 472.

8. Fisher, R. A., Statistical Methods for Research Workers, 6th ed. Edinburgh, Oliver and Boyd, 1936, page 166.

9. Shetlar, M. R., Schmidt, H. L., Jr., Lincoln, R. B., deVore, J. K., Bullock, J. A., and Hellbaum, A. A., Response of the serum polysaccharide fractions and protein fractions following cortisone treatment of patients with rheumatic fever. J. Lab. \& Clin. Med., 1952, 39, 372.

10. Weimer, H. E., and Redlich-Moshin, J., Comparative effects of intramuscular injections of ACTH, cortisone, and saline on serum glycoprotein levels. Proc. Soc. Exper. Biol. \& Med., 1953, 84, 34.

11. Shetlar, M. R., and Shetlar, C. L., Effect of cortisone on serum glycoprotein and seromucoid levels of rats. Proc. Soc. Exper. Biol. \& Med., 1955, 88, 622.

12. Greenspan, E. M., Survey of clinical significance of serum mucoprotein level. Arch. Int. Med., 1954, 93, 863.

13. Greenspan, E. M., Lehman, I., Graff, M. M:, and Schoenbach, E. B., Comparative study of the serum glycoproteins in patients with parenchymatous hepatic disease or metastatic neoplasia. Cancer, 1951, 4, 972.

14. Berkman, J., Rifkin, H., and Ross, G., The serum polysaccharides in diabetic patients with and without degenerative vascular disease. J. Clin. Invest., 1953, 32, 415.

15. Shetlar, M. R., Payne, R. W., Bullock, J. A., Patrick, D. R., Hellbaum, A. A., and Ishmael, W. K., Comparative studies of serum polysaccharides in rheumatoid arthritis and degenerative joint disease. J. Clin. Invest., 1953, 32, 1208.

16. Seibert, F. B., Pfaff, M. L., and Seibert, M. V., Serum polysaccharide in tuberculosis and carcinoma. Arch. Biochem., 1948, 18, 279.

17. Shetlar, M. R., Shetlar, C. L., Richmond, V., and Everett, M. R., The polysaccharide content of serum fractions in carcinoma, arthritis and infections. Cancer Research, 1950, 10, 681.

18. Bollett, A. J., The hexosamine content of the serum globulins in normal and pathological sera. J. Clin. Invest., 1957, 36, 51.

19. Winzler, R. J., and Smyth, I. M., Studies on the mucoproteins of human plasma. II. Plasma mucoprotein levels in cancer patients. J. Clin. Invest., 1948, 27, 617.

20. Levy, L., and Jackson, R. L., Studies on the mucoproteins of blood (abstract). J. Lab. \& Clin. Med., 1951, 38, 921. 
21. Seibert, F. B., Nelson, J. W., and Seibert, M. V., Correlation of extent of tuberculosis with amount of polysaccharide in the serum. Proc. Soc. Exper. Biol. \& Med., 1943, 52, 219.

22. Seibert, F. B., Seibert, M. V., Atno, A. J., and Campbell, H. W., Variation in protein and polysaccharide content of sera in the chronic diseases, tuberculosis, sarcoidosis and carcinoma. J. Clin. Invest., 1947, 26, 90.

23. Lustig, B., and Langer, A., Uber die Bestimmung und den Gehalt am freiem, Eiweiss und gebundenem Nichteiweisszucker im normalen und pathologischen Serum, Liquor und Harn. Biochem. Ztschr., 1931, 242,320 .

24. Shetlar, M. R., Foster, J. V., Kelley, K. H., Shetlar, C. L., Bryan, R. S., and Everett, M. R., The serum polysaccharide level in malignancy and in other pathological conditions. Cancer Research, 1949, 9, 515.

25. Hinsberg, $K$., and Merten, R., Der gebundene Zucker im Blutserum bei verschiedenen Krankheiten, inbesondere beim Carcinom. Ztschr. klin. Med., 1938, 135, 76.

26. Bierry, H., Sucre protéidique et protéides sanguines a l'état normal et pathologique. Compt. rend. Acad. d. sc., 1937, 204, 1681.

27. Stary, von Z., Akkurt, S., and Bodur, H., Untersuchungen uber den Polysaccharidegehalt von Blutserum und Pleuraexsudaten bei der Lungentuberkulose. Med. Monatschr., 1954, 11, 722.

28. Stary, von Z., Kaleoğlu, O., and Bursa, F., Uber die Hyperpolysaccharidaemie und ihre Klinischen Formen. Istanbul Contributions to Clinical Science, 1951, 1, 158.

29. Shetlar, M. R., Kelly, K. H., Foster, J. V., Shetlar, C. L., and Everett, M. R., Serum polysaccharide levels in pregnancy, parturition and post-partum state. Am. J. Obst. \& Gynec., 1950, 59, 1140.
30. Sachs, B. A., Cady, P., and Ross, G., An abnormal lipid-like material and carbohydrate in the sera of patients with multiple myeloma. Am. J. Med., 1954, 17, 662.

31. Blix, G., Tiselius, A., and Svensson, H., Lipids and polysaccharides in electrophoretically separated blood serum proteins. J. Biol. Chem., 1941, 137, 485.

32. Payne, R. W., Shetlar, M. R., Bullock, J. A., Patrick, D. R., Hellbaum, A. A., and Ishmael, W. K., The serum polysaccharide protein ratio $(P R)$ as a measure of rheumatoid arthritis activity. Ann. Int. Med., 1954, 41, 775.

33. Nilsson, I., Über den Glucosamingehalt des Blutserums bei gesunden und Pneumoniekranken. Biochem. Ztschr., 1937, 291, 254.

34. West, R., and Clarke, D. H., The concentration of glucosamine in normal and pathological sera. J. Clin. Invest., 1938, 17, 173.

35. Boas, N. F., and Soffer, L. J., The effect of adrenocorticotropic hormone and cortisone on the serum hexosamine level in acute disseminated lupus erythematosus. J. Clin. Endocrinol., 1951, 11, 39.

36. Boas, N. F., and Reiner, M., Effect of ACTH and cortisone on serum hexosamine and gammaglobulin levels in acute disseminated lupus erythematosus. J. Clin. Endocrinol., 1951, 11, 890.

37. Simkin, B., Bergman, H. C., and Prinzmetal, M., Studies on coronary circiulation V. Quantitative changes in a serum mucoprotein following the occurrence of myocardial infarction. Am. J. Med., 1949, 6, 734.

38. Kelley, V. C., Adams, F. H., and Good, R. A., Serum mucoproteins in patients with rheumatic fever. Pediatrics, 1953, 12, 607. 